\title{
Vacuoles: a hollow threat?
}

\author{
Tony L. Yaksh, PhD
}

Published online: 7 January 2010

(C) The Author(s) 2010. This article is published with open access at Springerlink.com

In this issue of the Journal, Bawolak et al. ${ }^{1}$ focus their studies on a mechanism that provides a provocative insight into the complexity of systems that organize the intracellular environment. This mechanism may also serve to describe the effects produced by agents that are applied in high concentrations to the outer membrane of the cell... in this case, local anesthetics. As the authors note, aside from their block of sodium channels in excitable membranes, local anesthetics are known to have many "off target" effects. The authors discuss a mechanism that reflects upon the effect of local anesthetics, specifically procaine and lidocaine, in forming intracellular vacuoles and the role of a specific enzyme in vacuolar (V)-ATPase in producing this phenomena. I comment on this paper from the perspective of the potential significance of this vacuolar mechanism, not only from the perspective of the smooth muscle cell that they employ but from the point of view of the potential effects of this mechanism on issues resulting from the effects of local anesthetics on nerves, the typical intended target systems for which these agents are most commonly employed.

The clinically relevant phenomenon of "conduction block" produced by cocaine was revealed in the late 19th century by the topical delivery of that agent to tissues such as the cornea or by delivery directly to the peripheral nerve or spinal cord using the hypodermic needle and syringe. Recognizing the limitations and side effects posed by the drug, early pharmaceutical chemistry led to the synthesis of

T. L. Yaksh, PhD (ه)

Departments of Anesthesiology and Pharmacology, University of California, San Diego, Anesthesia Research Lab (MC 0818), 9500 Gilman Drive, La Jolla, California 92093, USA

e-mail: tyaksh@ucsd.edu a variety of analogues with an early appreciation that distinguishing elements of the structure activity relationship were the amino ester and amino amide linkages which form a weakly basic structure. ${ }^{2}$

\section{Local anesthetic toxicity}

From the earliest days, incidences of neurological symptoms after neuraxial delivery have been reported, but these indicators were often considered minimal and/or reflecting a misadventure with the injection. Since the early 1990s, however, local anesthetics were reported as being associated with cauda equina syndrome, which was taken to reflect damage to S2-S4 sensory roots after continuous spinal anesthesia. ${ }^{3} \mathrm{~A}$ variant on that syndrome is a transient sensory dysesthesia that involves injury limited to lower lumbar roots (transient radicular irritation). ${ }^{4}$ These problems have been observed in patients receiving several agents, and they appear to be associated with local concentration. It is important to note that the incidence of radiculopathies was evidently augmented when the local anesthetics were delivered though microbore intrathecal catheters. Given the poor redistribution demonstrated from these catheters, an important aspect in considering the role of concentration in this observed lesion is the likelihood that the local anesthetic tended to remain locally and coat the adjacent roots. ${ }^{3}$ These human observations led to preclinical work in rats where it was shown that a variety of local anesthetics, including lidocaine, bupivacaine, and tetracaine, produced a concentration-induced hind limb motor disability. Histologic examination revealed white matter axonal degeneration, vacuolization in grey matter, infiltration of macrophages, and degeneration of Schwann cell sheaths. ${ }^{5}$ 


\section{Molecular mechanisms}

The molecular mechanism of this toxicity has been the subject of great debate. Failure to repeat these effects with tetrodotoxin suggests the effects are not due to simple sodium channel block. Various mechanisms have been proposed and reflect events observed with a variety of agents at concentrations believed to occur after local application. These events, reviewed elsewhere, include disruption of blood nerve barrier, decreased perineural blood flow, mitochondrial damage, inhibition of axonal transport, and increases in intracellular ionized calcium that triggered both necrosis and apoptosis. ${ }^{4,6}$ The panoply of these effects and the $\mathrm{mmol} \cdot \mathrm{L}^{-1}$ concentrations involved in their appearance after in vitro and in vivo exposure have led to the appreciation that the effects, unlike sodium channel block, may represent a broad impact on cellular morphology and function. Thus, it has been argued that these molecules may form micelles at the high concentrations employed, and such micelles may yield a detergentlike disruptive effect on the lipid membranes. ${ }^{7}$ A second possibility may be their ability to form intracellular vacuoles.

\section{Local anesthetics and vacuole formation}

Local anesthetics are weakly basic amines $(\mathrm{pKa} \geq 7)$ that become deprotonated in the extracellular space, diffuse across the membrane to become reprotonated in the more acidic intracellular space, and thus recover their ability to block the pore of the voltage-gated sodium channel. Closer examination reveals the situation to be even more complex. It is now appreciated that intracellular compartments are rendered acidic by the proton pump vacuolar (V)-ATPase. Here, the basic amine becomes reprotonated and trapped. This accumulation of trapped molecules results in the osmotic formation of large membraneless intracellular vacuoles. These phenomena have been observed in a variety of weakly basic molecules, such as ammonia, aminopyridines, and antihistiminics and, in the present experiments, with procaine and lidocaine. ${ }^{1}$ Importantly, a pivotal role of V-ATPase in this trapping and vacuole formation is suggested by the ability of the V-ATPase inhibitor, bafilomycin A1, to prevent the formation of vacuoles. While the present studies employed primary smooth muscle cells, consistent with the ubiquity of the presence and activity of v-ATPase, such drug-evoked vacuolization has been demonstrated in many cell systems, including dorsal root ganglion cells. ${ }^{8}$

\section{Functional consequences of this vacuole formation}

Among the many issues, three main issues come to mind:

1) These acidic vacuoles lead to a sequestration of the protonated molecule. This impacts on the local $\mathrm{pK}$ of the agent as it increases the apparent intracellular volume of distribution and potentially reduces the acute bioavailability of the agent though the sequestering. Alternatively, the sequestering results in an intracellular drug store available for release and initiation of an extended duration of action. ${ }^{9}$ The latter is true if the drug is not subject to intravacuolar metabolism.

2) The formation of the vacuoles and their trapping of the drug will lead to local increases in concentration in the intracellular pools to which local organelles may be exposed and lead to toxicity. The present experiments were carried out with concentrations of up to $5 \mathrm{mmol} \cdot \mathrm{L}^{-1}$, which induced strong vacuolar responses but had no effect on mitochondrial function or cell death. In other cell systems, including neuronal, a variety of local anesthetics caused neurite retraction and caspase activation, including lidocaine and procaine at concentrations of $5-10 \mathrm{mmol} \cdot \mathrm{L}^{-1}$ (see for example $\left.{ }^{10}\right)$. It would have been interesting to determine if the block of V-ATPase would have shifted the caspase activation and cell death curve to the right.

3) Vacuoles can result in the inhibition of the axonal transport of organelles, particularly along restricted pathways such as provided by neurites. ${ }^{8}$ Persistent changes in axonal transport and vacuolation may cause cellular dysfunction and degeneration. Where examined, it is interesting to note that impairment of the movement of organelles and degeneration of distal dendrites are common observations associated with local anesthetic actions. In this situation, it might be speculated that the deleterious role of the vacuoles may be dependent on the restricted structure provided by the neurite and, accordingly, would not be seen in systems such as a smooth muscle cells. Further, one could speculate that this process should be relevant wherever a neuron is exposed to membrane-permeable weak bases.

\section{Future directions}

The study of Bawolak et al. ${ }^{1}$ was clearly provocative and well done, although limited. The assessment of the broader applicability of this mechanism depends on the development of a broader structure activity relationship than that 
provided by procaine and lidocaine. While they were chosen for being representatives of molecules with amide and ester linkages, they differ remarkably in their $\log \mathrm{P}$. This difference makes it difficult to compare mechanisms, particularly in terms of their being sequestered. It will be interesting to look at other anesthetics of the same linkage class matched for lipid partition. Such studies would allow direct comparisons with other published reports regarding the vacuolar forming properties of these molecules with their known effects on cell survival, apoptosis, and necrosis. Further, this group has spent considerable time examining smooth muscle cells; while this may be an "off target" action of local anesthetics, one would hope these studies will be extended to Schwann cells and DRG, where local anesthetic pathology has also been specifically identified. These observations suggest that there are a number of future directions that might be pursued.

1) It seems reasonable that the pathology associated with vacuoles may be dependent on the cell morphology. It will be interesting to learn if indeed vacuoles can impair transport of organelles in different structural systems. Accordingly, does lidocaine or bupivacaine reduce trafficking of specific markers in the axon or proximal to the dendrites? If so, is this blocked by inhibitors of V-ATPase?

2) The phenomena of vacuolization raise interesting issues with regard to the role played by these structures in local drug pharmacokinetics. Given the concentration gradients involved, they may be particularly important for any systems in which the drug is locally/topically applied, e.g., topical, perineural, or intrathecal delivery. It is self-evident that an important line of research will be to pursue the effects of V-ATPase inhibition on local anesthetic kinetics and duration of action. It will be particularly interesting to determine the local concentrations of target drugs in these vacuoles as a function of time after topical application and whether vacuolar structures indeed provide a reservoir for ongoing drug release.

3) It will be intriguing to determine if V-ATPase blockade will enhance the anesthetic potency of local anesthetics in perineural or intrathecal models. Given previous work on organ systems ${ }^{11}$, presumably such potentiation would be most evident in the most lipophilic compounds, for example, the octanol/buffer partition coefficients for several anesthetics are: bupivacaine (560), ropivacaine (115), lidocaine (110), mepivacaine (42), chloroprocaine (17.4), and Procaine (3.1). ${ }^{12}$

4) If the vacuoles formed by weak base local anesthetics play a role in local toxicity, then the evident study will be to examine the effects of inhibitors of V-ATPase on local toxicity. Many models that exist to address this question have been validated for the peripheral nerve ${ }^{13}$ and for the intrathecal space. ${ }^{14}$ Conversely, if pathology is associated with small weakly basic amine molecules, it should be noted that one would predict other small weakly basic agents to have parallel effects after perineural or intrathecal delivery.

Whether vacuoles themselves play a pivotal role in the pathology of local anesthetics remains to be seen, but the work of Bawolak et al. suggests many potentially fruitful directions of research. The issue of local anesthetic toxicity is not a new one. It has been almost 100 years since Wossidlo reported that $5 \%$ procaine resulted in changes in the Nissl staining of dog DRG. ${ }^{15}$ One wonders behind which bubble the answer lies.

\section{Les vacuoles: une menace en l'air?}

Dans ce numéro du Journal, les études de Bawolak et coll. ${ }^{1}$ se concentrent sur un mécanisme qui propose de nouvelles pistes concernant la complexité des systèmes qui organisent l'environnement intracellulaire. Ce mécanisme peut également servir à décrire les effets produits par des médicaments appliqués à de fortes concentrations sur la membrane externe de la cellule-en l'occurrence, les anesthésiques locaux. Comme les auteurs le soulignent, on sait que les anesthésiques locaux ont plusieurs effets « hors cible » outre leur capacité à bloquer les canaux sodiques des membranes excitables. Le mécanisme présenté ici reflète l'effet des anesthésiques locaux, plus spécifiquement de la procaïne et de la lidocaïne, en formant des vacuoles intracellulaires. Les auteurs décrivent également le rôle d'une enzyme spécifique à l'ATPase vacuolaire (ATPase-V) qui provoque ce phénomène. Mon commentaire traite de la signification potentielle de ce mécanisme vacuolaire, non seulement du point de vue de la cellule du muscle lisse que les auteurs examinent, mais également du point de vue des effets potentiels de ce mécanisme sur les complications causées par les anesthésiques locaux au niveau des nerfs, soit les systèmes traditionnellement ciblés et pour lesquels ces agents sont le plus fréquemment utilisés.

Le phénomène pertinent d'un point de vue clinique du «bloc de conduction » provoqué par la cocaïne a été découvert à la fin du $19^{\mathrm{e}}$ siècle par la libération topique de cet agent à des tissus tels que la cornée, ou l'administration directe du produit au nerf périphérique ou à la moelle épinière à l'aide d'une aiguille hypodermique et d'une seringue. Consciente des limites et des effets secondaires du médicament, la chimie pharmaceutique a, à ses débuts, synthétisé plusieurs composés analogues, tout en réalisant rapidement que les liaisons amino-ester 
et amino-amide, qui forment une structure faiblement basique, constituaient des éléments distinctifs de la relation structure-activité. ${ }^{2}$

\section{Toxicité des anesthésiques locaux}

On a rapporté l'incidence de symptômes neurologiques dès les débuts de l'administration neuraxiale. Toutefois, ces événements ont souvent été considérés comme minimes et/ou le reflet d'une mésaventure lors de l'injection. Depuis le début des années 1990, il a été observé que les anesthésiques locaux étaient associés au syndrome de la queue de cheval, lequel a été considéré comme le reflet d'une atteinte aux racines sensitives S2-S4 à la suite d'une rachianesthésie continue. ${ }^{3}$ Parmi les variantes de ce syndrome, notons une dysesthésie sensitive transitoire impliquant une lésion qui se limite aux racines lombaires inférieures (irritation radiculaire transitoire). ${ }^{4}$ On a observé ces problèmes chez les patients recevant plusieurs agents, et ils semblent associés à la concentration locale. Il est important de noter que l'incidence des radiculopathies a augmenté de façon évidente lorsque les agents anesthésiques étaient administrés par le biais de sondes intrathécales microbores. La mauvaise redistribution de ces sondes ayant été démontrée, un élément important lorsqu'on examine le rôle de la concentration dans cette lésion est la probabilité que l'anesthésique local a eu tendance à rester au même endroit et enduire les racines adjacentes. ${ }^{3}$ Ces observations chez l'humain ont poussé des chercheurs à mener des études précliniques chez le rat, lesquelles ont démontré que divers anesthésiques locaux, y compris la lidocaïne, la bupivacaïne et la tétracaïne, produisaient un handicap moteur de la patte arrière produit par la concentration. L'examen histologique a révélé une dégénérescence axonale de la substance blanche, la formation de vacuoles dans la matière grise, l'infiltration de macrophages et la dégénérescence des gaines des cellules de Schwann. ${ }^{5}$

\section{Mécanismes moléculaires}

Le mécanisme moléculaire de cette toxicité a fait l'objet de nombreuses discussions. Le fait que ces effets ne puissent pas être reproduits avec de la tétrodotoxine laisse supposer que ces effets ne sont pas provoqués par le simple bloc des canaux sodiques. Divers mécanismes ont été proposés et se fondent sur les effets observés avec toutes sortes d'agents à des concentrations atteintes, selon toute vraisemblance, après l'application locale. Ces événements, passés en revue ailleurs dans la littérature, comprennent la rupture de la barrière hématonerveuse, la diminution du flux sanguin périneural, une lésion mitochondriale, l'inhibition du transport axonal, ainsi que des augmentations du calcium ionisé intracellulaire, provoquant nécrose et apoptose. ${ }^{4,6} \mathrm{La}$ panoplie de ces effets et les concentrations en $\mathrm{mmol} \cdot \mathrm{L}^{-1}$ nécessaires à leur apparition après une exposition in vitro et in vivo ont mené à la supposition que ces effets, au contraire du bloc des canaux sodiques, pourraient avoir de vastes répercussions sur la morphologie et la fonction cellulaires. Dès lors, il a été avancé que ces molécules pourraient former des micelles aux concentrations élevées employées, et que de telles micelles pourraient avoir un effet perturbateur similaire à celui d'un détergent sur les membranes lipidiques. ${ }^{7}$ Une autre possibilité serait leur capacité à former des vacuoles intracellulaires.

\section{Les anesthésiques locaux et la formation de vacuoles}

Les anesthésiques locaux sont des amines faiblement basiques ( $\mathrm{pKa} \geq 7$ ) qui perdent leurs protons dans l'espace extracellulaire, diffusent à travers la membrane pour redevenir protonés dans l'espace intracellulaire, plus acide, récupérant ainsi leur capacité à bloquer le pore du canal sodique voltage-dépendant. Un examen approfondi révèle que la situation est bien plus complexe. On sait désormais que les compartiments intracellulaires deviennent acides en raison de l'ATPase-(V) vacuolaire de la pompe à protons. Ici, l'amine basique devient reprotoné et est retenu. Cette accumulation de molécules retenues provoque la formation par osmose de larges vacuoles intracellulaires sans membrane. Ces phénomènes ont été observés avec plusieurs molécules faiblement basiques telles que l'ammoniaque, les aminopyridines et les anti-histaminiques et, dans les expériences de Bawolak et coll., la procaïne et la lidocaïne. ${ }^{1}$ Il importe de noter que l'ATPase-V pourrait jouer un rôle prépondérant dans cet emprisonnement et la formation de vacuoles, comme le suggère la capacité de l'inhibiteur de 1'ATPase-V, la bafilomycine A1, à prévenir la formation de vacuoles. Bien que les études en question ici aient utilisé des cellules primaires du muscle lisse en raison de l'omniprésence et de l'activité répandue de l'ATPase-V dans ces cellules, une telle vacuolisation d'origine médicamenteuse a été observée dans de nombreux systèmes cellulaires, notamment dans les cellules ganglionnaires de la racine dorsale. ${ }^{8}$

\section{Conséquences fonctionnelles de cette formation de vacuoles}

Parmi les nombreux problèmes provoqués par la formation de vacuoles, trois problèmes principaux viennent à l'esprit : 
1) Ces vacuoles acides provoquent une séquestration de la molécule protonée. Cela a un impact sur le pK local de l'agent étant donné que cela augmente le volume intracellulaire apparent de distribution et réduit potentiellement la biodisponibilité aiguë de l'agent par séquestration. En revanche, la séquestration a pour résultat un entreposage intracellulaire du médicament, lequel peut ensuite être libéré pour produire une action prolongée, ${ }^{9}$ en autant que le médicament n'est pas métabolisé dans la vacuole.

2) La formation des vacuoles et leur 'emprisonnement' provoqueront des augmentations locales de la concentration du médicament dans les bassins intracellulaires, concentration à laquelle les organelles locales pourraient être exposées, causant ainsi une toxicité. Les expériences en question ici ont été réalisées avec des concentrations allant jusqu'à $5 \mathrm{mmol} \cdot \mathrm{L}^{-1}$, ce qui a provoqué d'importantes réponses vacuolaires mais n'a pas eu d'effet sur la fonction mitochondriale ou la mort cellulaire. Dans d'autres systèmes cellulaires, notamment les systèmes neuronaux, divers anesthésiques locaux ont causé une rétraction des neurites et l'activation des caspases, notamment la lidocaïne et la procaïne à des concentrations de $5-10 \mathrm{mmol} \cdot \mathrm{L}^{-1}$ (voir par exemple $\left.{ }^{10}\right)$. Il aurait été intéressant de voir si le bloc de l'ATPase-V aurait déplacé l'activation des caspases et la courbe de mort cellulaire vers la droite.

3) Les vacuoles peuvent provoquer l'inhibition du transport axonal d'organelles, tout particulièrement le long de voies restreintes telles que celles offertes par les neurites. ${ }^{8}$ Des changements permanents au niveau du transport axonal et de la vacuolisation pourraient provoquer un dysfonctionnement et une dégénérescence cellulaires. Lorsque cela fait l'objet d'une analyse, il est intéressant de noter que la détérioration du mouvement des organelles et la dégénération des dendrites distaux sont des observations qu'on associe souvent aux actions des anesthésiques locaux. Dans un tel cas, on peut supposer que le rôle délétère des vacuoles pourrait dépendre de la nature restreinte procurée par le neurite et, ainsi, ne serait pas observé dans des systèmes tels que les cellules du muscle lisse. En outre, on peut supposer que ce processus surviendrait partout où un neurone est exposé à des bases faibles perméables aux membranes.

\section{Perspectives d'avenir}

L'étude de Bawolak et coll. ${ }^{1}$ est clairement provocatrice et bien construite, bien que limitée. L'évaluation de l'applicabilité plus large de ce mécanisme dépend du développement d'une relation structure-activité plus large que celle offerte par la procaïne et la lidocaïne. Ces agents ont été sélectionnés en raison du fait qu'ils sont représentatifs des molécules avec des liaisons amide et ester, mais leur $\log \mathrm{P}$ est considérablement différent. En raison de cette différence, il est difficile de comparer les mécanismes, particulièrement en termes de leur séquestration. Il sera intéressant d'observer d'autres anesthésiques de la même classe de liaison et appariés pour leur partition lipidique. Dans de telles études, il serait possible de réaliser des comparaisons directes avec d'autres comptes-rendus publiés portant sur les propriétés de formation de vacuoles de ces molécules et leurs effets connus sur la survie cellulaire, l'apoptose et la nécrose. En outre, Bawolak et coll. ont passé un temps considérable à examiner les cellules du muscle lisse; bien qu'il pourrait s'agir d'une action « hors cible » des anesthésiques locaux, il est à espérer que ces études seront étendues pour inclure les cellules de Schwann et les cellules ganglionnaires de la racine dorsale, deux environnements où la pathologie de l'anesthésique local a également été mise en exergue. Ces observations suggèrent que plusieurs pistes futures pourraient être explorées.

1) Il semble sensé que la pathologie associée aux vacuoles soit dépendante de la morphologie de la cellule. Il sera intéressant de voir si les vacuoles peuvent vraiment entraver le transport des organelles dans divers systèmes structurels. De la même façon, la lidocaïne et la bupivacaïne réduisent-elles le trafic de marqueurs spécifiques dans l'axone ou près des dendrites? Le cas échéant, cette action est-elle bloquée par les inhibiteurs de l'ATPase-V?

2) Les phénomènes de vacuolisation soulèvent des questions intéressantes quant au rôle que ces structures jouent dans la pharmacocinétique des anesthésiques locaux. Étant donné les gradients de concentration nécessaires ici, ces phénomènes pourraient être particulièrement importants pour tout système dans lequel l'application du médicament est locale/topique, par ex. l'injection topique, périneurale ou intrathécale. Il va sans dire qu'une ligne de recherche importante sera d'explorer les effets de l'inhibition de l'ATPase-V sur la cinétique et la durée d'action des anesthésiques locaux. Il sera particulièrement intéressant de déterminer les concentrations locales de médicaments cibles dans ces vacuoles en fonction du temps après l'application topique et de voir si les structures vacuolaires fournissent effectivement un réservoir pour une libération prolongée du médicament.

3) Il serait intéressant de voir si le bloc de l'ATPase-V augmente la puissance anesthésique des anesthésiques locaux dans des modèles périneuraux et intrathécaux. Si l'on se fonde sur les travaux antérieurs portant sur 
les systèmes des $\operatorname{organes}^{11}$, il est à supposer qu'une telle potentialisation serait la plus évidente pour les molécules les plus fortement lipophiles. Par exemple, les coefficients de partage octanol/ tampon de divers anesthésiques sont : bupivacaïne (560), ropivacaïne (115), lidocaïne (110), mépivacaïne (42), chloroprocaïne $(17,4)$, et procaïne $(3,1){ }^{12}$

4) Si les vacuoles formées par des anesthésiques locaux faiblement basiques jouent un rôle dans la toxicité locale, alors l'étude évidente sera d'évaluer les effets des inhibiteurs de l'ATPase-V sur la toxicité locale. Nombre des modèles existants pour aborder cette question ont été validés pour le nerf périphérique ${ }^{13}$ et l'espace intrathécal. ${ }^{14}$ Inversement, si la pathologie est associée à de petites molécules d'amines faiblement basiques, il est à noter qu'on pourrait déduire que d'autres petits agents faiblement basiques auraient des effets parallèles après une libération périneurale ou intrathécale.

Il reste à voir si les vacuoles jouent en soi un rôle clé dans la pathologie des anesthésiques locaux, mais les travaux de Bawolak et coll. offrent plusieurs orientations de recherche potentiellement fructueuses. La question de la toxicité des anesthésiques locaux ne date pas d'hier. Presque 100 ans se sont écoulés depuis que Wossidlo a rapporté que de la procaïne $5 \%$ provoquait des changements dans la coloration de Nissl de cellules ganglionnaires de la racine dorsale chez le chien. ${ }^{15}$ Il est à se demander derrière quelle bulle la réponse se cache.

Conflicts of interest None declared.

Open Access This article is distributed under the terms of the Creative Commons Attribution Noncommercial License which permits any noncommercial use, distribution, and reproduction in any medium, provided the original author(s) and source are credited.

\section{References}

1. Bawolak MT, Morissette G, Marceau F. Vacuolar ATPasemediated sequestration of local anesthetics in swollen macroautophagosomes. Can J Anesth 2010; 57(3).
2. Ruetsch YA, Boni T, Borgeat A. From cocaine to ropivacaine: the history of local anesthetic drugs. Curr Top Med Chem 2001; 1 : 175-82.

3. Drasner K. Lidocaine spinal anesthesia: a vanishing therapeutic index? Anesthesiology 1997; 87: 469-72.

4. Pollock JE. Neurotoxicity of intrathecal local anaesthetics and transient neurological symptoms. Best Pract Res Clin Anaesthesiol 2003; 17: 471-84.

5. Sakura S, Kirihara Y, Muguruma T, Kishimoto T, Saito $Y$. The comparative neurotoxicity of intrathecal lidocaine and bupivacaine in rats. Anesth Analg 2005; 101: 541-7.

6. Hogan $Q H$. Pathophysiology of peripheral nerve injury during regional anesthesia. Reg Anesth Pain Med 2008; 33: 435-41.

7. Kitagawa N, Oda M, Totoki T. Possible mechanism of irreversible nerve injury caused by local anesthetics: detergent properties of local anesthetics and membrane disruption. Anesthesiology 2004; 100: 962-7.

8. Hiruma H, Kawakami T. Effects of 4-aminopyridine on organelle movement in cultured mouse dorsal root ganglion neurites. J Mol Neurosci 2009. DOI:10.1007/s12031-009-9219-2.

9. Morissette G, Bouthillier J, Marceau F. Trapping of adrenergic decongestant drugs into cellular endomembrane compartments: toxicological and pharmacological consequences. Int Immunopharmacol 2007; 7: 1869-79.

10. Perez-Castro R, Patel S, Garavito-Aguilar ZV, et al. Cytotoxicity of local anesthetics in human neuronal cells. Anesth Analg 2009; 108: 997-1007.

11. Morissette G, Lodge R, Bouthillier J, Marceau F. Receptorindependent, vacuolar ATPase-mediated cellular uptake of histamine receptor-1 ligands: possible origin of pharmacological distortions and side effects. Toxicol Appl Pharmacol 2008; 15: 320-31.

12. Strichartz GR, Sanchez V, Arthur GR, Chafetz R, Martin D. Fundamental properties of local anesthetics. II. Measured octanol:buffer partition coefficients and $\mathrm{pKa}$ values of clinically used drugs. Anesth Analg 1990; 71: 158-70.

13. Myers RR, Kalichman MW, Reisner LS, Powell HC. Neurotoxicity of local anesthetics: altered perineurial permeability, edema, and nerve fiber injury. Anesthesiology 1986; 64: 29-35.

14. Sakura S, Hashimoto $K$, Bollen AW, Ciriales $R$, Drasner $K$. Intrathecal catheterization in the rat. Improved technique for morphologic analysis of drug-induced injury. Anesthesiology 1996; 85: 1184-9.

15. Wossidlo E. Experimentelle untersuchungen uber veranderungen Nissl'schen granulma bei der lumbalanasthesie. Arch f Klin Chir 1908; 86: 1017-53. 